Observation of relativistic effects in collective Thomson scattering

J. S. Ross, S. H. Glenzer, J. P. Palastro, B. B. Pollock, D. Price, L. Divol, G. R. Tynan, D. H. Froula

October 28, 2009

Physical Review Letters 
This document was prepared as an account of work sponsored by an agency of the United States government. Neither the United States government nor Lawrence Livermore National Security, LLC, nor any of their employees makes any warranty, expressed or implied, or assumes any legal liability or responsibility for the accuracy, completeness, or usefulness of any information, apparatus, product, or process disclosed, or represents that its use would not infringe privately owned rights. Reference herein to any specific commercial product, process, or service by trade name, trademark, manufacturer, or otherwise does not necessarily constitute or imply its endorsement, recommendation, or favoring by the United States government or Lawrence Livermore National Security, LLC. The views and opinions of authors expressed herein do not necessarily state or reflect those of the United States government or Lawrence Livermore National Security, LLC, and shall not be used for advertising or product endorsement purposes. 


\title{
Observation of relativistic effects in collective Thomson scattering
}

\author{
J. S. $\operatorname{Ross}^{1,2}$, S. H. Glenzer ${ }^{1}$, J. P. Palastro ${ }^{1}$, B. B. Pollock ${ }^{1,2}$, \\ D. Price ${ }^{1}$, L. Divol ${ }^{1}$, G. R. Tynan ${ }^{2}$, and D. H. Froula ${ }^{1}$ \\ ${ }^{1}$ Lawrence Livermore National Laboratory, \\ P.O. Box 808, Livermore, California 94551 and \\ ${ }^{2}$ Mechanical and Aerospace Engineering Department, \\ University of California at San Diego, \\ 9500 Gilman Drive, La Jolla, CA 92093-0411
}

\begin{abstract}
We observe relativistic modifications to the Thomson scattering spectrum in a traditionally classical regime: $v_{o s c} / c=e E_{0} / c m \omega_{0}<<1$ and $T_{e}<1 \mathrm{keV}$. The modifications result from scattering off electron-plasma fluctuations with relativistic phase velocities. Normalized phase velocities $v / c$ between 0.03 and 0.12 have been achieved in a $\mathrm{N}_{2}$ gas-jet plasma by varying the plasma density from $3 \times 10^{18} \mathrm{~cm}^{-3}$ to $7 \times 10^{19} \mathrm{~cm}^{-3}$ and electron temperature between $85 \mathrm{eV}$ and $700 \mathrm{eV}$. For these conditions, the complete temporally resolved Thomson scattering spectrum including the electron and ion features has been measured. A fully relativistic treatment of the Thomson scattering form factor has been developed and shows excellent agreement with the experimental data.
\end{abstract}


Thomson scattering [1-3] is an important diagnostic for determining the plasma conditions in laser-produced [4-6], tokamak [7-9], and pinch [10, 11] plasmas. Each of these systems can be vulnerable to plasma instabilities which are a strong function of the plasma conditions. In particular, inertial confinement fusion (ICF) target designs for the National Ignition Facility (NIF) [12] require detailed understanding of the temperature and density in order to mitigate laser-plasma instabilities [13]. The high-density, high-temperature conditions in ICF targets present a new regime for collective Thomson scattering where understanding relativistic effects are essential.

Collective Thomson scattering is the scattering of light from electron-density fluctuations with a wave vector $\mathbf{k}$ whose inverse is larger than the Debye length $\left(k \lambda_{D}<1\right)$. Therefore, collective scattering probes fluctuations corresponding to the natural modes of the plasma and the spectra contain four distinct features, corresponding to ion-acoustic and electronplasma resonances. Light scattered from the co- and counter propagating modes is red- and blue-shifted by the frequency of the resonance, and the intensity of the scattered light is in general governed by the Landau damping of each mode. Therefore, the frequency and shape of the scattered light can be an accurate measure of the plasma parameters. A majority of laser-plasma experiments [4-6] have resolved the ion-acoustic feature to provide an accurate measure of the electron temperature and scattering from the electron-plasma resonance is ideal for measuring the electron density, but has seen limited application in the collective regime due to the small scattering cross section.

For non-collective Thomson scattering $\left(k \lambda_{D}>1\right)$, where the light is scattered by free electrons, relativistic effects governed by the thermal velocity of the electrons $[16,17]$ have been observed [18]. Relativistic nonlinear effects due to extremely high Thomson scattering probe intensities $\left(>10^{18} \mathrm{~W} \mathrm{~cm}^{-2}\right.$ ) causing relativistic electron motion have been measured [15]. Previous collective-scattering measurements from electron-plasma waves [14] have only resolved the red-shifted resonance, making characterization of relativistic effects difficult. There have been no definitive measurements of relativistic effects from thermal fluctuations in the collective Thomson scattering regime using a low intensity probe.

In this Letter, we present the first observation of a relativistic correction to the collective Thomson scattering spectrum. We measure a factor of 1.9 difference between the peak power measured in the blue-shifted electron-plasma wave resonance and the peak power calculated using the non-relativistic Thomson scattering form factor for a normalized phase velocity of 
$\beta=\omega / k c=0.12$. This result is attributed to two effects: (1) the relativistic aberration of light which causes a source that emits uniformly in the rest frame to preferentially emit in its direction of motion when moving relativistically, and (2) the interaction of the initial electron motion with the magnetic field of the Thomson scattering probe beam. Both of these effects change the shape of the scattering spectrum by increasing (decreasing) the power scattered into the blue-shifted (red-shifted) electron-plasma feature and are governed by the phase velocity of the plasma wave which can be relativistic even at low electron temperatures. As the phase velocity is reduced, the relativistic correction is reduced, but is always significant for our measurements made between $\beta=0.03$ and $\beta=0.12$. A fully relativistic treatment of the scattering spectrum in the weak probe limit has been developed [20] that shows excellent agreement with our measurements. An analysis of the scattering spectrum expected from ICF plasmas is performed and shows the potential error in inferred plasma conditions using a non-relativistic scattering spectrum. Furthermore, the ion-acoustic features are simultaneously measured, and no relativistic effects at the low temperatures studied are observed or expected.

The experiment was performed at the Jupiter Laser Facility using the Janus Laser System. A $300 \mathrm{~J}, 527 \mathrm{~nm}(2 \omega)$, laser is focused at target chamber center (TCC) using an $f / 6.7$ lens [Fig 1(a)]. A continuous phase plate is used to produce a 600- $\mu \mathrm{m}$ super Gaussian focal spot. The pulse length is a 3-ns long plateau with a $150 \mathrm{ps}$ rise and fall. A 1.5-mm diameter gas jet with a nitrogen backing pressure ranging from 10 to 400 psi positioned $1.0 \mathrm{~mm}$ below TCC provides neutral gas densities between $1.4 \times 10^{18} \mathrm{~cm}^{-3}$ and $1 \times 10^{19} \mathrm{~cm}^{-3}$.

An $f / 4$ collection lens collimated light scattered $90^{\circ}$ relative to the laser beam from the Thomson scattering volume located at TCC. The scattered light is split using a 532 $\mathrm{nm}$ notch filter and propagated to a pair of spectrometers. The notch filter reflectes light with a wavelength of $532 \mathrm{~nm} \pm 10 \mathrm{~nm}$ which is focused onto the entrance slit of a 1-meter spectrometer using an $f / 10$ focusing lens. The light transmitted through the notch filter was focused onto the entrance slit of a $1 / 3$-meter spectrometer using an $f / 10$ spherical focusing mirror. Both optical systems provide a magnification of 2.5. The 1-meter and $1 / 3$-meter spectrometers are coupled to an S-20 and S-1 Hamamatsu streak camera respectively. Both

systems use a spectrometer entrance slit of $200 \mu \mathrm{m}$ and a streak camera entrance slit of $400 \mu \mathrm{m}$. A 2400 grooves/mm grating in the 1-meter system results in a spectral resolution of $0.056 \mathrm{~nm}$ and a temporal resolution of $550 \mathrm{ps}$ [19]. The $1 / 3$-meter system uses a 150 


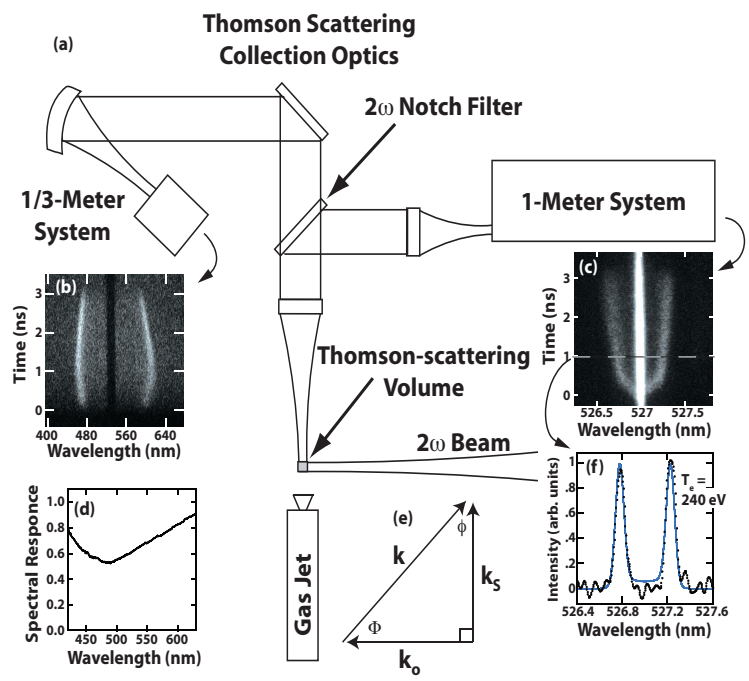

FIG. 1: (a) Experimental setup. Thomson scattered light is collected $90^{\circ}$ relative to the laser beam. Scattering from (b) electron-plasma waves and (c) ion-acoustic waves are displayed. (d) The spectral response of the system used to measure the electron-plasma wave spectra is characterized using a tungsten lamp. (e) The Thomson scattering k-vector diagram shows the orientation of the k-vector that is probed. (f) The ion-acoustic wave spectrum (dots) at $1 \mathrm{~ns}$ is fit by the calculated form factor (solid line) with an electron temperature of $240 \mathrm{eV}$ and a density of $1.4 \times 10^{19} \mathrm{~cm}^{-3}$ after subtracting the background and stray light.

grooves/mm grating resulting in a spectral resolution of $3.6 \mathrm{~nm}$ and a temporal resolution of 400 ps. The Thomson scattering volume $600 \mu \mathrm{m}$ x $160 \mu \mathrm{m}$ x $80 \mu \mathrm{m}$ is defined by the overlap of the spectrometer and streak camera slit images at TCC with the laser beam. A spectrally calibrated Tungsten lamp inserted at TCC is used to measure the spectral intensity response of the 1/3-meter system [Fig. 1(d)]. A maximum spectral sensitivity variation of $60 \%$ is observed over the $200 \mathrm{~nm}$ measurement region.

The plasma temperature has been well characterized by fitting the ion-acoustic spectra [Fig. 1(f)] with the fully relativistic form factor. The electron temperature increases rapidly over the first $100 \mathrm{ps}$ and plateaus at a nearly constant temperature. At low densities $\left(3 \times 10^{18}\right.$ $\left.\mathrm{cm}^{-3}\right)$, the electron temperature plateaus at around $90 \mathrm{eV}$ while at high densities $\left(7 \times 10^{19}\right.$ $\mathrm{cm}^{-3}$ ) the temperature peaks around $700 \mathrm{eV}$. The uncertainty in these measurements is better than $15 \%$ and is dominated by our uncertainties in the ion temperature.

Figure 2 shows the temporally resolved collective Thomson scattering from electron- 


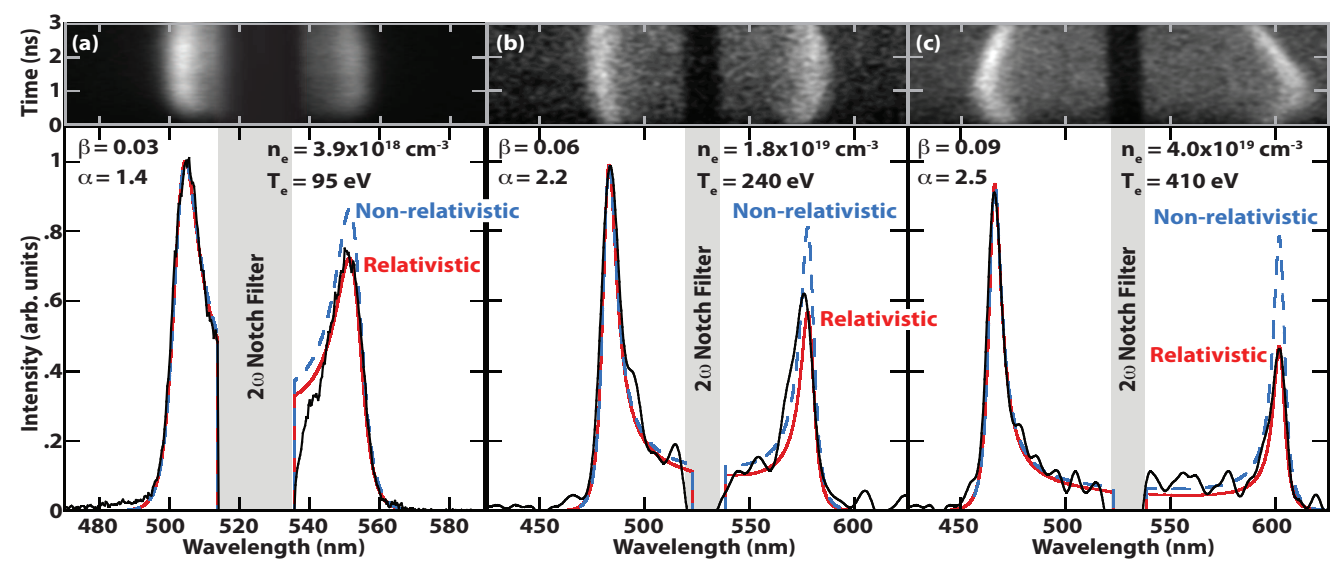

FIG. 2: Top panels: raw streak data. Bottom panels: experimental data (solid-black line) at 2 ns normalized to the blue-shifted feature and compared to the non-relativistic (blue-dashed line) and relativistic (solid-red line) form factors. As the temperature and density increase, the phase velocity of the electron-plasma wave increases (a) $\beta=0.03$, (b) $\beta=0.06$, (c) $\beta=0.09$ and the difference between the non-relativistic and relativistic form factors becomes more pronounced.

plasma waves for various phase velocities (i.e. densities). The primary difference between the non-relativistic and relativistic form factors is readily observable in the reduction in the red-shifted electron-plasma wave peak. As the normalized phase velocity of the electronplasma wave increases from 0.03 to 0.09 , the relativistic effects become more pronounced.

A fully relativistic treatment has been developed [20] and approaches, for our low temperature conditions, a second order treatment including terms of order $\beta^{2}$. The form factor shows excellent agreement with the experimental data (Fig. 2). In the case where $\left(v_{t h} / c\right)^{2}<<1$, scattering is measured perpendicular to the incident electric field direction, and $\beta^{3}<<1$, the second order treatment can be written $\mathrm{P}(k, \omega)=\mathrm{P}_{e}(k, \omega)+\mathrm{P}_{i}(k, \omega)$, where

$$
\begin{array}{r}
\mathrm{P}_{e}(k, \omega)=\frac{\mathrm{P}_{0} r_{e}^{2} n_{e}}{2 \mathrm{~A} c k} f_{e 0}(\beta)\left\{\left[\left(1+\frac{\omega}{\omega_{i}}\right)^{2}-\beta^{2}\right.\right. \\
\left.+\zeta\left(\frac{\omega}{\omega_{i}}\right) \beta\right]\left|\frac{1-\chi_{e}}{\varepsilon}\right|^{2}+\zeta\left(\frac{\omega}{\omega_{i}}\right)\left|\frac{\chi_{e}}{\varepsilon}\right|^{2} \beta \\
\left.+\beta^{2}\left(\frac{\omega_{p}}{\omega}\right)^{2} \operatorname{Re}\left[\frac{1+\chi_{i}}{|\varepsilon|^{2}}\right]-\frac{1}{2} \beta^{2}\right\},
\end{array}
$$




$$
\begin{gathered}
\mathrm{P}_{i}(k, \omega)=\frac{Z \mathrm{P}_{0} r_{e}^{2} n_{e}}{2 \mathrm{~A} c k} f_{i 0}(\beta)\left\{\left[\left(1+\frac{\omega}{\omega_{i}}\right)^{2}-\beta^{2}\right.\right. \\
\left.\left.\quad+2 \zeta\left(\frac{\omega}{\omega_{i}}\right) \beta\right]\left|\frac{\chi_{e}}{\varepsilon}\right|^{2}+\beta^{2}\left(\frac{\omega_{p}}{\omega}\right)^{2} \operatorname{Re}\left[\frac{\chi_{e}}{|\varepsilon|^{2}}\right]\right\},
\end{gathered}
$$

$\mathrm{P}_{0}$ is the incident power, $\mathrm{A}$ is the cross-sectional area of the Thomson scattering volume, $r_{e}$ is the classical electron radius, $n_{e}$ is the electron density, $\varepsilon=1+\chi_{e}+\chi_{i}$ is the dielectric function, $\chi_{e}, \chi_{i}$ is the electron, ion susceptibility respectively, $Z$ is the average ionization state, $f_{e 0}(\beta)$ and $f_{i 0}(\beta)$ are the one-dimensional Maxwellian distributions for electrons and ions respectively, $\omega=\omega_{s}-\omega_{i}, \omega_{s}$ is the scattered frequency, $\omega_{i}$ is the incident frequency, $\zeta=\left(\hat{k} \cdot \hat{k}_{s}\right)$, and $\hat{k}_{s}$ is the unit vector from the source to the detector.

For our conditions, the relativistic effects are observed in the asymmetric scattering spectrum and attributed to two effects. The first effect is due to the relativistic aberration, also referred to as the relativistic "headlight" effect, where light is preferentially directed in the emitter's direction of propagation [21]. The second effect is a result of the electron motion in the direction of the incident light vector interacting with the magnetic field of the Thomson scattering probe laser. The resulting $\vec{v} \times \vec{B}$ force, which is neglected in the non-relativistic treatment, is in the same direction as the force of the incident electric field. When the electron is moving towards the detector, the increased force on the electron enhances the scattered power. When the electron is moving away from the detector the force is in the opposite direction and the scattered power is reduced. The effects of these corrections can be estimated by taking the ratio of the peak power in the blue- and red-shifted electron-plasma wave resonances,

$$
\frac{\mathrm{P}^{\text {blue }}}{\mathrm{P}^{\text {red }}} \approx \underbrace{\frac{\mathrm{P}_{\mathrm{nr}}^{\text {blue }}}{\mathrm{P}_{\mathrm{nr}}^{\text {nr }}}}_{A} \underbrace{\frac{(1+\beta \cos \phi)^{2}}{(1-\beta \cos \phi)^{2}}}_{B} \underbrace{\frac{(1+\beta \cos \Phi)^{2}}{(1-\beta \cos \Phi)^{2}}}_{C},
$$

where $\phi$ is the angle between $\hat{k}$ and $\hat{k}_{S}, \Phi$ is the angle between $\hat{k}$ and $\hat{k}_{0}$, both of which are shown in Fig. 1(e) and equal $45^{\circ}$ for our scattering geometry, and

$$
\mathrm{P}_{\mathrm{nr}}(k, \omega)=\frac{\mathrm{P}_{0} r_{e}^{2} n_{e}}{2 \mathrm{~A} c k}\left[\left|\frac{1+\chi_{i}}{\varepsilon}\right|^{2} f_{e 0}(\beta)+Z\left|\frac{\chi_{e}}{\varepsilon}\right|^{2} f_{i 0}(\beta)\right],
$$

is the non-relativistic Thomson scattered power spectrum [1-3]. Eq. (3) is recovered from Eq. (1) by letting terms of order $\beta^{2}=\omega / \omega_{i}=0$. The asymmetry in term $A$ of Eq. (2) is due to Landau damping, term $B$ is due to relativistic aberration, and term $C$ is due to the initial particle motion interacting with the magnetic field. 


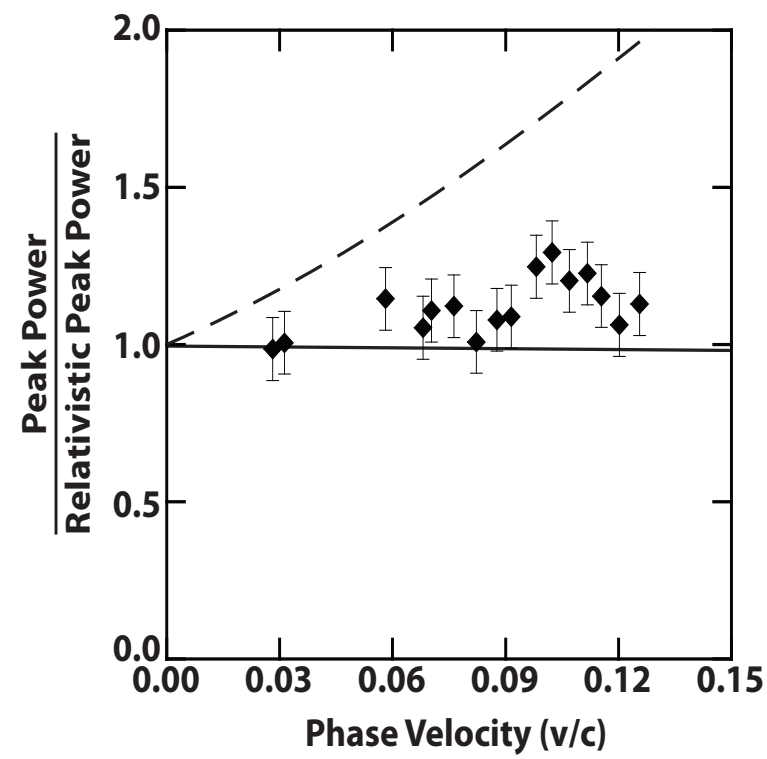

FIG. 3: The measured peak powers in the red-shifted feature are divided by the calculated peak powers [Eq. (1), diamonds] and are plotted as a function of phase velocity. The electron temperature ranged from $85 \mathrm{eV}$ to $720 \mathrm{eV}$. Data in perfect agreement with the relativistic form factor would have a value of unity. The ratio of the non-relativistic peak power (dashed line) and the corrected non-relativistic peak power [Eq. (2), solid line] divided by the relativistic peak power are shown.

Figure 3 shows good agreement between the experimental data and the form factor including relativistic corrections [Eq. (1)] for normalized phase velocities ranging from 0.03 to 0.12 . The data is compared with calculations using the non-relativisitc power spectrum [Eq. (3)] where the asymmetry in the peaks is given by the Landau damping. When using the simple model to correct for the relativistic effects [Eq. (2)], the scattered power agrees with the fully relativistic form factor. The spectra are normalized to the peak power in the blue-shifted feature (see Fig. 2) and fit using the relativistic form factor. The measured peak scattered power in the red-shifted feature divided by the peak scattered power calculated using the relativistic form factor is plotted in Fig. 3. Thomson scattered light is observed for the full 3-ns duration of the laser beam (Fig. 2) allowing the electron temperature and density to be determined at 250 ps intervals by fitting Eq. 1 to the measured spectrum. The measured spectra are obtained by integrating over a 200 ps region. For clarity we average multiple shots at a particular phase velocity; the error bars represent the standard deviation within this average. 


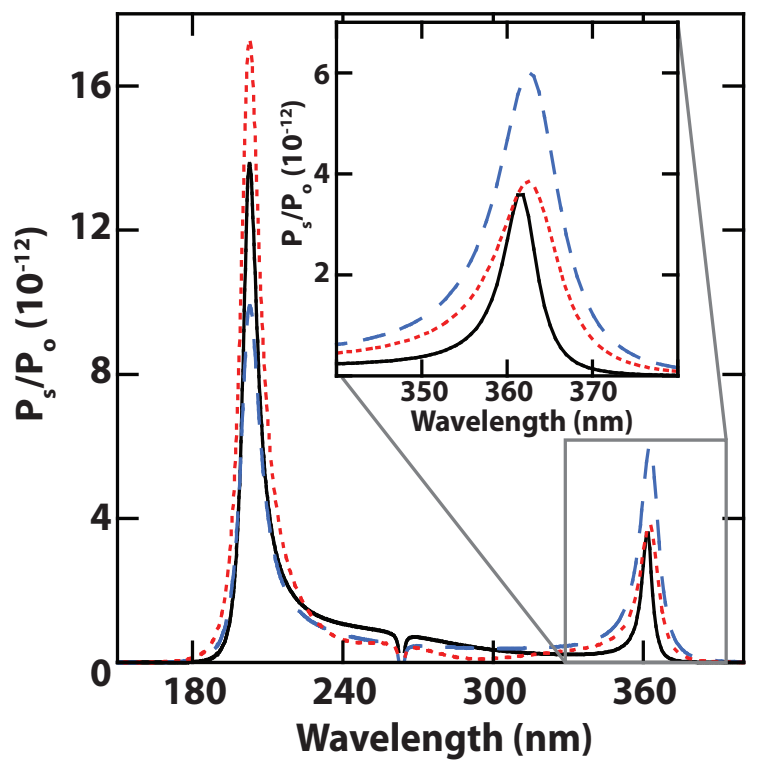

FIG. 4: The non-relativistic [Eq. (3), blue-dashed line], second order relativistic [Eq. (1), reddotted line] and fully relativistic (solid-black line) form factors are compared for typical plasma conditions on the NIF: an electron temperature of $10 \mathrm{keV}$, electron density of $8.0 \times 10^{20} \mathrm{~cm}^{-3}$, and a scattering angle of 35 degrees using a Thomson scattering probe beam with a wavelength of 263.5 nm. These conditions result in a normalized electron-plasma wave phase velocity of 0.37 .

The normalized phase velocity $(\beta=\omega / k c)$ of the electron-plasma wave is calculated from the plasma parameters, the scattering angle, and the incident laser wavelength. Maximizing Eq. (1) for $\beta$ provides the normalized phase velocity of the local maxima corresponding to the electron-plasma wave resonances. All normalized phase velocities reported in this Letter refer to the normalized phase velocity of the red-shifted electron-plasma wave feature.

Fitting the ion-acoustic spectrum with Eqs. (1) and (3) shows that relativistic corrections are not important for scattering from the ion-acoustic resonances at our conditions. Discrepancies are not expected until the phase velocity of the ion-acoustic wave is greater than one percent of the speed of light which is estimated to be when $T_{e} \approx 140 \mathrm{keV}$ assuming $Z T_{e}>>3 T_{i}$ and a fully ionized nitrogen plasma.

Figure 4 compares the non-relativistic form factor [Eq. (3)], a relativistic form factor including terms of second order in $\beta$ [Eq. (1)], and the fully relativistic form factor for typical NIF conditions. Notice that for collective high-temperature conditions the asymmetry between the different form factors is pronounced and a $2 \mathrm{~nm}$ wavelength shift resulting from the relativistic maxwellian distribution used to evaluate the fully relativistic form factor is 
observed. Neglecting the relativistic frequency shift produces a $13 \%$ error in the measured electron temperature and a $5 \%$ error in the measured electron density for the conditions shown in Fig. 4.

In conclusion, we have measured relativistic effects in collective Thomson scattering from electron-plasma waves which are attributed to the relativistic "headlight" effect and the electron motion in the direction of the incident light vector interacting with the magnetic field of the Thomson scattering probe. A fully relativistic form factor is presented and shows excellent agreement with the measured spectra. We show that a relativistic form factor is required to accurately analyze scattering from electron-plasma waves in most laser produced plasmas where $T_{e}$ and $n_{e}$ are greater than $100 \mathrm{eV}$ and $1.0 \times 10^{19} \mathrm{~cm}^{-3}$ respectively.

I would like to acknowledge contributions from J. Sheffield and C. Clayton as well as private communications with Zheng, et. al. regarding the importance of first-order relativistic corrections to the Thomson scattering form factor. This work was performed under the auspices of the U.S. Department of Energy by Lawrence Livermore National Laboratory under Contract DE-AC52-07NA27344 and was partially funded by the Laboratory Directed Research and Development Program under project tracking code 08-LW-070.

[1] J. A. Fejer, Canadian Journal of Physics 38, 1114 (1960).

[2] J. Sheffield, Plasma Scattering of Electromagnetic Radiation (Academic, New York, 1975).

[3] I. H. Hutchinson, Principles of Plasma Diagnostics (Cambridge, 1987).

[4] S. H. Glenzer, et. al., Phys Rev Lett79, 1277 (1997).

[5] D. H. Froula, et. al., Phys Rev Lett 88, 105003 (2002).

[6] D. H. Froula, et al., Physics of Plasmas 10, 1846 (2003).

[7] N. Peacock, et. al., Nature 224, 488 (1969).

[8] R. Behn, et. al., Phys Rev Lett 62, 2833 (1989).

[9] F. Alladio et. al., Phys Lett A 60, 39 (1977).

[10] T. Wrubel, et. al., Plasma Phys Contr F 42, 519 (2000).

[11] R. Pasqualotto, et. al., Rev Sci Instrum 70, 1416 (1999).

[12] J. D. Lindl, et. al., Physics of Plasmas 11, 339 (2004).

[13] H. Baldis, et. al., Phys Rev Lett 80, 1900 (1998). 
[14] S. H. Glenzer, et. al., Phys Rev Lett 82, 97 (1999).

[15] S. Chen, et. al., Nature 396, 653 (1998).

[16] R. Pechacek and et. al., Physics of Fluids 10, 1688 (1967).

[17] H. Bindslev, Plasma Phys Contr F 33, 1775 (1991).

[18] M. Mattioli et. al., Plasma Phys Contr F 17, 165 (1975).

[19] A. Visco, et. al., (AIP, 2008), vol. 79, p. 10F545.

[20] J. P. Palastro, et. al., in preparation, Phys. Rev. E (2009).

[21] E. F. Taylor and J. A. Wheeler, Spacetime Physics (W. H. Freeman and Company, San Francisco and London, 1966). 